\title{
Lymphovascular Space Invasion is an Independent Risk Factor for Nodal Disease and Poor Outcomes in Endometrioid Endometrial Cancer
}

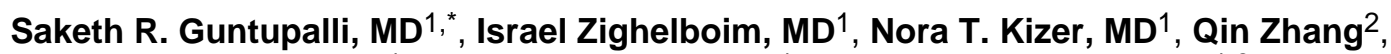 \\ Matthew A. Powell, MD ${ }^{1}$, Premal H. Thaker, MD ${ }^{1}$, Paul J. Goodfellow, PhD ${ }^{1,3}$, and David G. \\ Mutch, MD ${ }^{1}$ \\ ${ }^{1}$ Division of Gynecologic Oncology, Department of Obstetrics and Gynecology Washington, \\ University School of Medicine and Siteman Cancer Center, Saint Louis, MO 63110 \\ ${ }^{2}$ Division of Biostatistics, Washington University School of Medicine and Siteman Cancer Center, \\ Saint Louis, MO 63110 \\ ${ }^{3}$ Division of Endocrine and Oncologic Surgery, Washington University School of Medicine and \\ Siteman Cancer Center, Saint Louis, MO 63110
}

\begin{abstract}
Objective-Adjuvant radiotherapy improves local control but not survival in women with endometrial cancer. This benefit was shown in staged patients with "high intermediate risk" (HIR) disease. Other studies have challenged the need for systematic staging including lymphadenectomy. We sought to determine whether LVSI alone or in combination with other histologic factors predicts lymph node (LN) metastasis in patients with endometrioid endometrial cancer.
\end{abstract}

Methods-A retrospective review was conducted of patients with endometrioid endometrial carcinoma who had confirmed presence/absence of LVSI and clinicopathologic data necessary to identify HIR criteria. Kaplan-Meier curves were generated and univariate and multivariate analyses performed as appropriate.

Results-We identified 757 eligible patients and 628 underwent systematic lymphadenectomy for staging purposes. In the surgically staged group, 242 (38\%) patients met uterine HIR criteria and 196 (31\%) had LVSI. Both HIR and LVSI were significantly associated with LN metastasis. Among the HIR positive group, 59 had LN metastasis (OR 4.46, 95\% CI 2.72-7.32, $P<0.0001$ ). Sixty-six LVSI positive patients had nodal metastasis (OR 11.04, 95\% CI 6.39-19.07, $P<0.0001$ ). The NPV of LVSI and HIR negative specimens was $95.6 \%$ and $93.4 \%$ respectively. In multivariate analysis, PFS and OS were significantly reduced in both LVSI positive $(P<0.0001)$ and HIR patients $(P<0.0001)$ when compared to patients who were LVSI and HIR negative

Conclusions-HIR status and LVSI are highly associated with LN metastasis. These features are useful in assessing risk of metastatic disease and may serve as a surrogate for prediction of extrauterine disease.

\footnotetext{
"Corresponding Author: Saketh R. Guntupalli, MD, Department of Obstetrics and Gynecology, Division of Gynecologic Oncology, Washington University School of Medicine, 600 Euclid Street, Box 8400, St. Louis, MO 63108., Tel (314) 362-2003, Fax: (314) 362-2893, guntupallis@wudosis.wustl.edu.

The authors disclose no potential conflict of interest with regards to this work.
} 


\section{Introduction}

Endometrial cancer is the most common gynecologic malignancy in the United States with 43,000 new cases and 7,900 deaths in 2010 alone [1]. Eighty percent of women present at an early stage and thus overall 5-year survival rates approach 90-97\% [2, 3]. Systematic surgical staging including lymph node (LN) evaluation remains an important prognostic tool in women with endometrial cancer. Recently however, two large European studies have called into question the therapeutic value of routine lymph node sampling in patients afflicted with this disease. These studies evaluated 1,914 patients and found that survival was not different between patients who received lymphadenectomy and those who only underwent a total abdominal hysterectomy and removal of adnexae alone $[4,5]$. There is however, extensive controversy surrounding these results with regards to study design and inclusion criteria.

In the near future, uterine histopathologic features may become increasingly important and used as a surrogate risk marker and determinant of adjuvant therapy for patients with high risk disease. Several trials including PORTEC-1 and GOG protocol 99 have established factors such as age, grade, and depth of myometrial invasion as characteristics associated with increased risk for recurrence [2, 6]. Interestingly, these studies did not examine the association of these markers with lymph node metastasis, a significant predictor of outcome. LVSI in particular has been previously shown in a few, small scale studies to be a biomarker for both nodal disease and recurrence but the use of this finding remains controversial $[7,8]$. We hypothesized that LVSI may independently serve as a predictor of nodal disease in patients with endometrioid endometrial cancer in comparison to patients who meet other high risk criteria. The secondary aims of this study are the impact that LVSI has on overall and progression free survival in a cohort of nearly 800 patients.

\section{Materials and Methods}

Institutional Review Board approval was obtained for this retrospective study through the Human Research and Protection Office (HRPO). Our institution maintains a large database of prospectively gathered clinicopathologic data on patients treated for endometrial cancer. Both blood and tumor specimens were collected on all participating subjects at the time of surgery. The current study included patients diagnosed with endometrioid endometrial cancer over a 16-year period (1991-2007). This interval was chosen to allow for suitable follow-up for outcomes. Inclusion criteria were all women greater than 18 years of age with histological diagnosis consistent with endometrioid endometrial cancer. Women were excluded if outcome data were not available, if histology was other than endometrioid or if other synchronous cancers were present. The primary research question was whether LVSI alone or if in conjunction with HIR criteria ( LVSI, grade, outer myometrial invasion and age) is a predictor of nodal metastasis with secondary endpoints being the effect of these variables on overall and progression free survival.

All patients underwent total hysterectomy and removal of the uterine adnexae. In the staged cohort, pelvic lymph node dissection was accomplished by opening the retroperitoneal spaces along the external iliac vessels. Lymph node bearing tissue was removed with the boundaries of the dissection including the genitofemoral nerve laterally, the bifurcation of the common iliac vessels superiorly, the ureter medially and the deep circumflex iliac vein inferiorly. The obturator space below the external iliac vein was also identified and nodal tissue superior to the obturator nerve was excised. Para aortic nodes were removed bilaterally. The typical limits of the dissection were, inferiorly the bifurcation of the common iliac artery and superiorly the inferior mesenteric artery. Staging was assigned following the FIGO 1988 staging system for endometrial cancer [9]. 
Patients were deemed as high intermediate risk (HIR) for relapse based on the criteria defined by GOG protocol 99, conferring a $27 \%$ risk of relapse [6]. Specifically, patients were initially risk stratified by age as less than 50 years, between 50 and 70 years and greater than 70 years. Histologic criteria including the presence of lymphovascular space invasion, invasion of tumor into the outer one-third of the myometrium and grade 2-3 histology were used as markers for increased risk for recurrence. In the group less than 50 years all three criteria were required for patients to be deemed HIR; in the 50-70 aged group two factors and in the greater than 70 years group only one risk factor was required (Table $1)$.

Data on the presence or absence of lymphovascular space invasion (LVSI), tumor grade, histological type and depth of myometrial invasion was also abstracted from the pathology reports. All specimens were reviewed by experienced gynecologic pathologists. Lymphvascular space invasion was defined as the presence of adenocarcinoma, of any extent, in endothelium lined channels of uterine specimens extracted at the time of surgery, as described by GOG 99. Outer one-third myometrial invasion was defined as tumor presence in the outer one third of the uterus as measured from the innermost portion of the endometrial lining. Grade was defined by standard FIGO criteria. The cohort was analyzed according to presence or absence of LVSI as well as according to whether patients met GOG 99 high intermediate risk criteria.

Associations between LN metastasis, LVSI and HIR factors were assessed using Chi square tests in the 628 patients that underwent surgical staging. Multivariate analysis, utilizing logistic regression modeling, was used to evaluate the potential effects of co-variates including age, myometrial invasion, tumor grade and lymphovascular space invasion on lymph node metastasis. Patients who met HIR criteria were not included in the multivariate analysis as a separate group as these criteria incorporate LVSI (inclusion would be redundant due to the principal of multi-colinearity). Survival estimates were based on Kaplan-Meier calculations with overall survival (OS) defined as from date of surgery until date of death; patients were censored at last contact. Progression free survival (PFS) was defined as date of surgery until date of recurrence or progression. Cox proportional hazard models were fitted to independently evaluate the influence of co-variates (age, grade, myometrial invasion and LVSI). Hazard ratios for survival were found to be proportional up to 156 months follow-up and were included in ratio estimates. Effects were expressed as either an odds or hazard ratio with $95 \%$ confidence intervals as appropriate. Log-rank tests were used to examine the individual effects of variables on both overall and progression free survival. A $P$ value of $<0.05$ was considered statistically significant and all tests were 2sided unless otherwise indicated. Data analysis was performed using SAS 9.1 software (SAS Institutes, Cary, NC).

\section{Results}

A total of 1,083 patients were enrolled during the study period. Of these 143 were excluded due to lack of outcome data, 173 due to non-endometrioid histology and 10 due to the presence of a non-melanoma skin cancer, leaving a total of 757 for primary evaluation. One hundred twenty nine patients were excluded from analysis of $\mathrm{LN}$ metastasis because of lack or inadequate lymphadenectomy (reasons included clinically apparent advanced disease, large body-mass index (BMI) and/or technical inability to obtain lymph nodes) leaving 628 for analysis. Median follow-up time was 49.5 months (range 1-195 months). The demographics and clinicopathologic characteristics of the cohort are outlined in Table 2. Mean lymph node count was 18 and 5 for pelvic and paraaortic nodes respectively. In the surgically staged group, 242 (38\%) were classified as HIR and $196(31 \%)$ had evidence of LVSI. Within the HIR group, 156 (65\%) tumors were LVSI positive. Of the surgically 
staged patients, 66 LVSI positive patients and 59 HIR patients had LN metastasis. Univariate analysis revealed that LVSI (OR 11.04, 95\% CI 6.39-19.07; $P<0.0001$ ), and HIR criteria (OR 4.46, 95\% CI 2.72-7.32; $P<0.0001)$ were significantly associated with presence of LN metastasis. Multivariate analysis confirmed LVSI (OR 6.34, 95\% CI 3.45-11.66; $P<0.0001)$ and depth of myometrial invasion (OR 4.92, 95\% CI 2.82-8.59; $P<0.0001)$ as strong predictors of LN metastasis. The positive predictive value of LVSI and HIR criteria on LN metastasis was $33.6 \%$ and $24.2 \%$ respectively. The negative predictive value of absent LVSI and HIR factors on LN metastasis was $95.6 \%$ and $93.4 \%$ respectively.

The median progression free survival for the study cohort was 19 months (range 0-147 months). In univariate analysis both HIR criteria $(P<0.0001)$ or presence of LVSI alone $(P<$. $0001)$ were significantly associated with decreased overall survival. Multivariate analysis confirmed older age (HR 1.04 95\% CI 1.02-1.05, $P<0.0001$ ), higher grade (HR 1.61, 95\% CI $1.34-1.93, P<0.0001$ ), and LVSI (HR 1.98, 95\% CI 1.46-2.69; $P<0.0001$ ) to be independent predictors of decreased OS (Table 3). Univariate analysis also revealed HIR criteria $(P<0.0001)$ and presence of LVSI alone $(P<0.0001)$ to be associated with decreased PFS. Multivariate analysis showed older age (HR 1.03, 95\% CI 1.02-1.05; $P<0.0001$ ), grade (HR 1.59, 95\% CI 1.33-1.91; $P<0.0001$ ), outer one-third myometrial invasion (HR1.47, 95\% CI 1.09-1.97; $P=0.01$ ) and presence of LVSI (HR 2.14, 95\%CI 1.59-2.88; $P<0.0001$ ) to be associated with a decreased PFS (Table 3). Kaplan-Meier survival plots for OS and PFS were extrapolated for presence of LVSI and patients who met HIR criteria (Figures 12). In patients who were LVSI-positive (Figure 1-2), both OS and PFS were significantly reduced (log-rank test, $P<0.0001$ ). Patients who met GOG 99 HIR criteria (Figures 1-2) also showed decreased OS and PFS (log-rank test, $P<0.0001$ ).

\section{Discussion}

In the current study, we have shown that LVSI appears to be highly predictive of nodal disease in endometrial cancer. Further, LVSI was an independent predictor of both decreased overall and progression free survival. Previous studies evaluating LVSI have shown mixed results; Rasool et al examined 147 patients with endometrial cancer and found LVSI not to be predictive of recurrence or poor outcomes where as Gaducci et al found it to be associated with distant, hematogenous failures $[10,11]$. In a series of nearly 800 patients we have however found LVSI to be significantly associated with diminished survival. Histopathologic markers for recurrence and poor outcomes in endometrial cancer are becoming increasingly important in treatment planning. The PORTEC-1 trial established certain factors for recurrence in early stage patients including age, high grade histology and depth of myometrial tumor invasion [2]. Gynecologic Oncology Group (GOG) study 99 further validated this concept in a subgroup of patients who were deemed as "high intermediate risk" (HIR) for recurrence and decreased overall survival [6]. These patients had histologic features (LVSI, outer one-third myometrial invasion and FIGO grade 2-3 tumors) or combination of features which, stratified by age, put them at a significant risk for recurrence and decreased progression free survival. There was no difference in overall survival amongst HIR patients and those that did not meet these criteria when treated with radiotherapy, though the study itself was underpowered. Recurrence however, may have been mitigated by adjuvant radiotherapy in the HIR subset of patients.

Lymphadenectomy in endometrial cancer however remains highly controversial. The ASTEC study ( A Study in the Treatment of Endometrial Cancer) randomized nearly 1400 patients to TAH BSO with or without lymphadenectomy and found no survival advantage in patients undergoing the latter procedure [5]. Benedetti-Panici et al also validated this same concept in an additional cohort of 514 patients [4]. While these are both randomized controlled trials, they have inherent flaws. Benedetti et al's study is underpowered based on 
their own reported power calculations and number of patients enrolled. The extent of lymphadenectomy in the ASTEC trial was poor with only an average of 12 nodes taken at surgery. Chan et al have showed that the extent of lymphadenectomy was predictive of recurrence with 21-25 nodes being required for adequate nodal assessment [12]. Further para aortic nodes were not included in this study, a significant predictor of decreased PFS and OS. The ASTEC study also includes a disproportionate number of stage IA/IB patients who have a very low baseline risk of nodal disease, questioning the true power to detect a difference. Most importantly in the ASTEC trial patients with known lymph node metastasis were again randomized to the radiotherapy versus observation phase of the trial, disregarding the presence of nodal metastasis [13]. Conversely, lymphadenectomy has however been shown to be beneficial in multiple retrospective studies. Kilgore et al examined 649 patients, 205 who underwent full surgical staging with lymphadenectomy and found a survival benefit in early stage patients $(P=.02)$ [ 14]. Cragun et al further validated this in 509 patients with stage I-IIA endometrial cancer who underwent pelvic lymphadenectomy and found a survival benefit in grade 3 cancers[15]. Also, GOG 33 validated surgical staging with lymphadenectomy as nearly $25 \%$ of patients were ultimately upstaged after lymph node sampling was performed [16].

Despite this information, current data suggests that most endometrial cancers in the US are not treated by gynecologic oncologists and thus do not undergo full surgical staging with lymphadenectomy. Chan et al found that between 1999 and 2002 only $28 \%$ of these patients were treated by gynecologic oncologists [17]. Given these facts, biomarkers such as LVSI and HIR criteria for which patients may be at risk for occult nodal disease become increasingly important. Our data shows that the absence of LVSI has a NPV of 95\% and could therefore be used as a marker to risk-stratify patients with nodal disease. This is impressive and similar to the NPV of sentinel lymph node mapping in patients with malignant melanoma and vulvar cancer, obviating the need for further nodal dissection [18, 19]. This data is helpful in counseling patients who may undergo simple hysterectomy by a general gynecologist who is ultimately found to have an invasive cancer on final pathology. Since LVSI negative tumors would only confer a 5\% risk of nodal metastasis, these patients could be counseled for observation rather than proceeding with a restaging procedure.

Conversely, LVSI positive tumors have a high relative risk of nodal disease and may require further evaluation with lymphadenectomy and/or adjuvant radiotherapy and treatment of nodal basins. This data is also relevant in the triage of patients with early stage endometrial cancer that may not undergo systemic lymphadenectomy secondary to medical comorbidities including morbid obesity, pulmonary derangements and cardiac disease as these problems preclude lengthy operative times.

Strengths of our study include the large number of patients, pathologic review by a single team of pathologists, and adequate power to detect a statistically significant association between clinicopathologic variables and LN metastasis. Fully one-third of our patients met HIR criteria which is similar to the patients in GOG 99 and allows for a fair comparison between the two studies. Weaknesses include the innate bias common to retrospective analyses and inherent selection bias found at large tertiary referral centers such as ours. We also concede however that LVSI is difficult to assess during intraoperative frozen sectioning and is often reported on final pathological diagnosis. This study shows that LVSI positive tumors and patients that meet other HIR criteria are at significant risk for nodal metastasis. These data indicate that while lymphadenectomy provides important information, in an era of decreased surgical staging, features including LVSI may be used as a surrogate for future treatment planning. The negative predictive value of absent LVSI further helps to risk stratify patients for occult nodal disease. As we come to understand endometrial cancer better we have learned that the more information we have about an individual cancer, the better the treatment planning is for that individual's disease. Lymphadenectomy findings 
remain important in treatment planning and should be performed when feasible and safe. In patients unable to undergo lymphadenectomy, other prognostic variables may be used in the treatment planning process. LVSI, depth of invasion, grade, nodal involvement and age are all important predictors of both recurrence and survival and should be obtained when possible in the staging of patients with endometrial cancer.

\section{Acknowledgments}

Funding: Supported by RO1 CA71754 (P.J.G.) and Barnes-Jewish Foundation 00161-0806 (P.J.G.). The Siteman Cancer Center is supported by NCI Cancer Center Support Grant P30 CA91842.

\section{References}

1. Jemal A, Siegel R, Xu J, Ward E. Cancer statistics, 2010. CA Cancer J Clin. 2010; 60(5):277-300. [PubMed: 20610543]

2. Creutzberg CL, van Putten WL, Kopert PC, et al. Surgery and postoperative radiotherapy versus surgery alone for patients with stage 1 endometrial carcinoma: multicenter randomized trial. PORTEC study group. Post operative radiation Therapy in Endometrial Cancer Lancet. 2000; 355(9213):1404-1411.

3. Ries YJ, Gloeckler LA, LAG, Keel GE, Eisner MP, Lin YD, Horner M-J, et al. SEER survival monograph: cancer survival among adults: U.S. SEER program. :1988-2001.

4. Benedetti R, Panici P, et al. Systematic pelvic lymphadenectomy vs no lymphadenectomy in early stage endometrial carcinoma: a randomized clinical trial. J Natl Cancer Institute. 2008; 100:17071716.

5. Kitchener H, Swart AM, Qian Q, Amos C, Parmar MK, et al. Efficacy of systemic pelvic lymphadenectomy in early stage endometrial cancer (MRC ASTEC trial): A Randomized. Lancet. 2009; 373(9658):125-136. [PubMed: 19070889]

6. Keyes HM, Roberts JA, Brunetto VL, Zaino RJ, Spirtos NM, Bloss JF, Bell JG, et al. A phase III trial of surgery with or without adjunctive external pelvic radiation therapy in intermediate risk endometrial adenocarcinoma: A Gynecologic Oncology Group study. Gynecol Oncol. 2004; 94(1): 241-242.

7. Briet JM, Holemma H, Reesink N, Aalders JG, Mourits MJ, Nijman HW, et al. Lymphovascular space involvement: an independent prognostic factor in endometrial cancer. Gynecol Oncol. 2005; 96:799-804. [PubMed: 15721428]

8. Morrow CP, Bundy BN, Kurman RJ, Creasman WT, Heller P, Graham JE, et al. Relationship between surgical pathological risk factors and outcome in clinical stage I and II carcinoma of the endometrium: a Gynecologic Oncology Group study. Gynecol Oncol. 1999; 40(1):55-65. [PubMed: 1989916]

9. FIGO Corpus cancer staging. Int J Gynecol Obstet. 1989; 28:1990.

10. Rasool N, et al. Stage 1, grade 3 endometrioid adenocarcinoma of the endometrium: An analysis of clinical outcomes and patterns of recurrence. Gynecol Oncol. 2010; 116:10-14. [PubMed: 19875158]

11. Gadducci A, Cavazzana A, Cosio S, DI Cristofano C, Tana R, Genazzani AR, et al. Lymph vascular space involvement and outer one-third myometrial invasion are strong predictors of distant hematogenous failures in patients with stage I-II endometrioid-type endometrial cancer. Anticancer Res. 2009; 29(5):1715-1720. [PubMed: 19443392]

12. Chan JK, et al. Lymphadenectomy in endometrioid uterine cancer staging: how many lymph nodes are enough? A Study of 11,443 patients. Cancer. 2007; 109:2454-2460. [PubMed: 17503431]

13. Blake P, Swart AM, Orton J, Kitchener H, Whelan T, Lukka H, Eisenhauer E, Bacon M, Tu D, Parmar MK, Amos C, Murray C, Qian W. Adjuvant external beam radiotherapy in the treatment of endometrial cancer (MRC ASTEC and NCIC CTG EN.5 randomised trials): pooled trial results, systematic review, and meta-analysis. Lancet. 2009; 373(9658):137-146. [PubMed: 19070891] 
14. Kilgore LC, Partridge EE, Alvarez RD, Austin JM, Shingleton HM, Noojin F 3rd, Conner W. Adenocarcinoma of the endometrium: survival comparisons of patients with and without pelvic node sampling. Gynecol Oncol. 1995; 56(1):29-33. [PubMed: 7821843]

15. Cragun JM, Havrilesky LJ, Calingaert B, Synan I, Secord AA, Soper JT, Clarke-Pearson DL, Berchuck A. Retrospective analysis of selective lymphadenectomy in apparent early-stage endometrial cancer. J Clin Oncol. 2005; 23(16):3668-3675. [PubMed: 15738538]

16. Creasman WT, Morrow CP, Bundy BN, Homesley HD, Graham JE, Heller PB. Surgical pathologic spread patterns of endometrial cancer. A Gynecologic Oncology Group Study. Cancer. 1987 Oct 15; 60(8 Suppl):2035-2041. [PubMed: 3652025]

17. Chan JK, Sherman AE, Kapp DS, Zhang R, Osann KE, Maxwell L, Chen LM, Deshmukh H. Influence of gynecologic oncologists on the survival of patients with endometrial cancer. J Clin Oncol. 2011; 29(7):832-838. [PubMed: 21263082]

18. Valsecchi ME, Silbermins D, de Rosa N, Wong SL, Lyman GH. Lymphatic mapping and sentinel lymph node biopsy in patients with melanoma: a meta-analysis. J Clin Oncol. 2011; 29(11):14791487. [PubMed: 21383281]

19. de Hullu JA, Hollema H, Piers DA, Verheijen RH, van Diest PJ, Mourits MJ, Aalders JG, van Der Zee AG. Sentinel lymph node procedure is highly accurate in squamous cell carcinoma of the vulva. J Clin Oncol. 2000; 18(15):2811-2816. [PubMed: 10920128] 


\section{Research Highlights}

- LVSI is associated with nodal metastasis and poor survival in endometrial cancer.

- The NPV of 95\% with LVSI- negative tumors may exclude nodal disease.

- LVSI and other histological makers may be used as surrogates for treatment planning. 


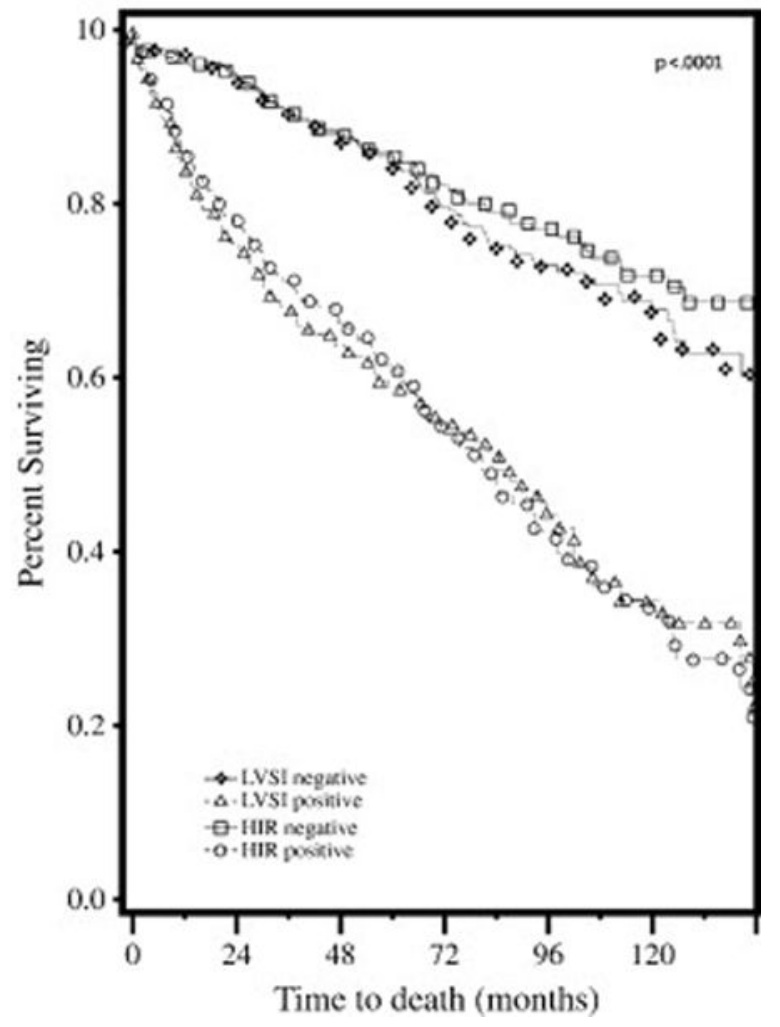

Figure 1.

Overall survival in LVSI-positive versus LVSI-negative patients and in patients who met HIR risk criteria versus HIR-negative patients. 


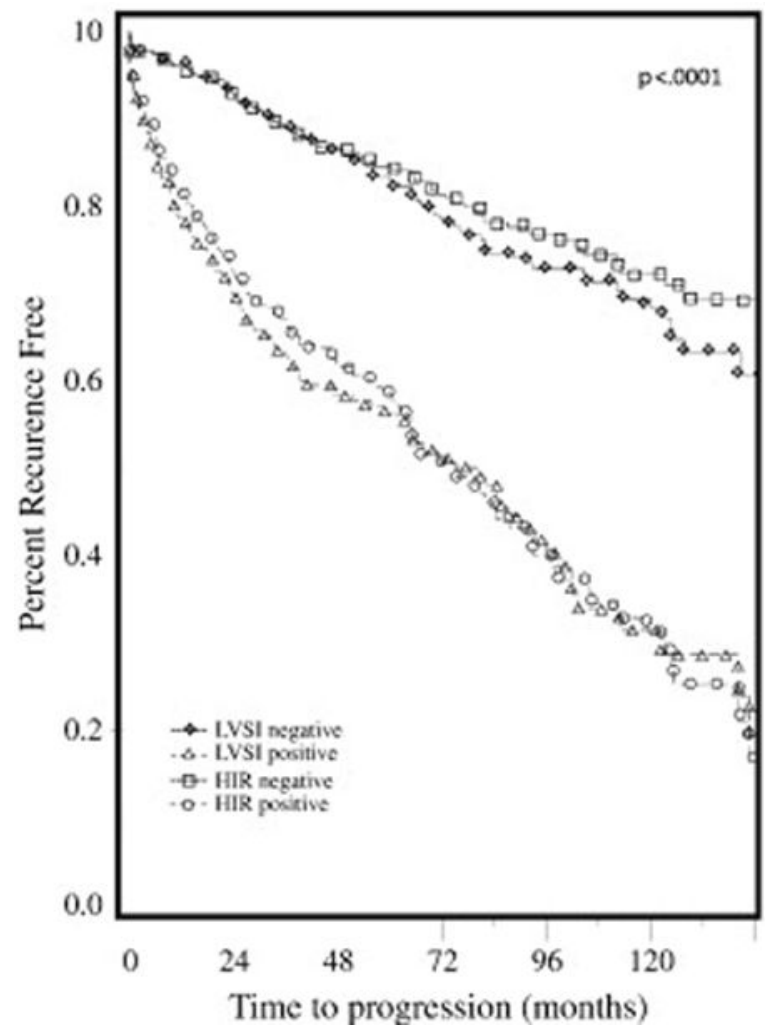

Figure 2.

Recurrence free survival in LVSI-positive versus LVSI-negative patients and in patients who met HIR risk criteria versus HIR-negative patients. 


\section{Table 1}

High Intermediate Risk (HIR) stratification per Gynecologic Oncology Group (GOG) Protocol \#99

\begin{tabular}{ll}
\hline Age divisions & \\
$-<50$ years & All 3 Criteria \\
$-50-70$ years & 2 criteria \\
$->70$ years & 1 criterion \\
Criteria & \\
-presence of lymphovascular space invasion (LVSI) \\
-invasion of tumor into outer one-third of myometrium \\
-grade 2 or 3 histology \\
\hline
\end{tabular}


Table 2

Clinicopathologic and Demographic Characteristics in 757 Endometrioid Endometrial Cancer Patients

\begin{tabular}{|l|l|}
\hline Stage * & \\
IA & $129(20.5 \%)$ \\
IB & $247(39.3 \%)$ \\
IC & $102(16.2 \%)$ \\
IIA & $19(3 \%)$ \\
IIB & $30(4.8 \%)$ \\
IIIA & $20(3.2 \%)$ \\
IIIC & $71(11.3 \%)$ \\
IVA & $2(0.3 \%)$ \\
IVB & $8(1.3 \%)$ \\
\hline Histological Grade & \\
1 (well differentiated) & $410(54.2 \%)$ \\
2 (moderately differentiated) & $232(30.6 \%)$ \\
3 (poorly differentiated) & $115(15.2 \%)$ \\
\hline Depth of myometrial invasion & $174(23 \%)$ \\
+ outer 1/3 involvement & $583(77 \%)$ \\
- outer 1/3 involvement & $239(31.6 \%)$ \\
\hline Lymphovascular Space Invasion (LVSI) \\
Present & $518(68.4 \%)$ \\
Absent & \\
\hline Age & $230(30 \%)$ \\
$<<\mathbf{5 0}$ & \\
$\mathbf{5 0 - 7 0}$ & \\
$>\mathbf{7 0}$ & \\
\hline
\end{tabular}

Stage information provided for the 628 surgically staged patients. 


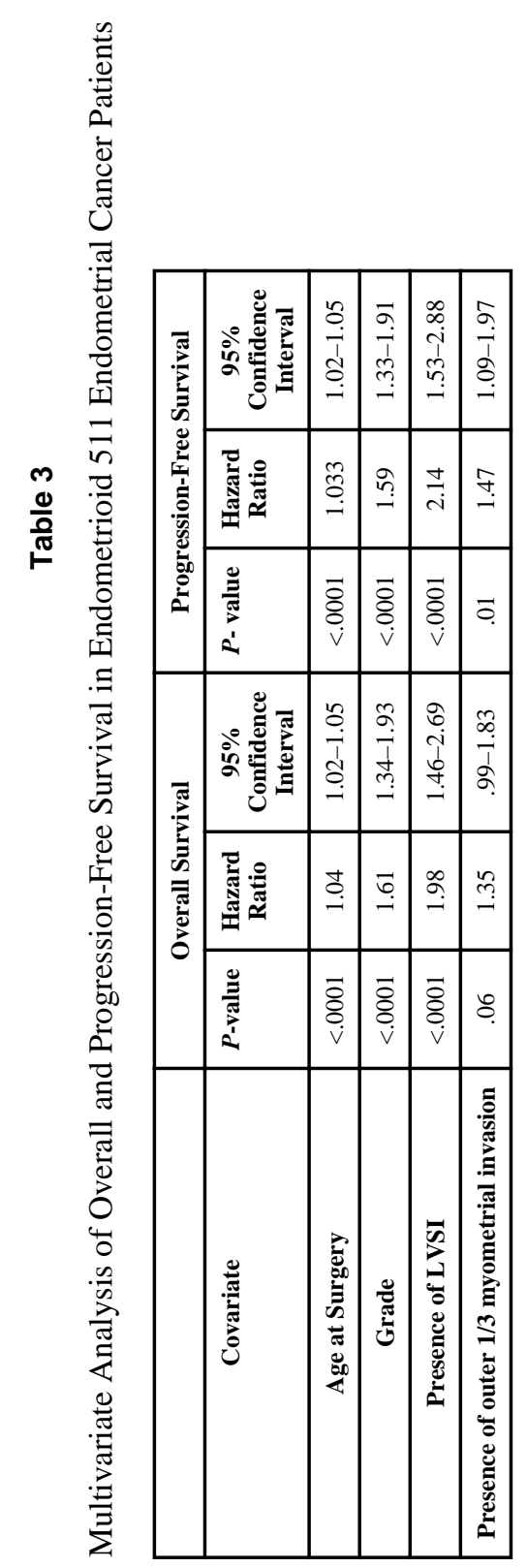

Gynecol Oncol. Author manuscript; available in PMC 2014 February 27. 